\title{
Preexisting Autoimmune Disease: Implications for Immune Checkpoint Inhibitor Therapy in Solid Tumors
}

Laura C. Kennedy, MD, PhD a,b; Shailender Bhatia, MDa,b; John A. Thompson, MDª,b; and Petros Grivas, MD, PhD

\section{ABSTRACT}

The use of immune checkpoint inhibitors ( $\mathrm{ICls}$ ) is rapidly expanding to the treatment of many cancer types, both in the metastatic setting and as an adjuvant to other therapies. Clinical trials using $\mathrm{ICls}$ have largely excluded patients with preexisting autoimmune diseases due to concerns for increased toxicity. However, emerging evidence shows that ICls may be considered in some patients with autoimmunity. This review discusses the commonalities between clinical autoimmune diseases and $\mathrm{ICl}$-induced immunotherapy-related adverse events, and summarizes the existing case series that describes patients with solid tumors who have a preexisting autoimmune disease. This review also discusses which patients with autoimmunity could be considered reasonable candidates for $\mathrm{ICl}$ therapy. J Natl Compr Canc Netw 2019;17(6):750-757 doi: $10.6004 /$ jnccn.2019.7310

${ }^{a}$ Department of Medicine, Division of Oncology, University of Washington, and ${ }^{b}$ Clinical Research Division, Fred Hutchinson Cancer Research Center, Seattle, Washington.

\section{Background}

Immune checkpoint inhibitors (ICIs) have dramatically altered cancer therapy. The ICI-class drugs include antiPD-1 (nivolumab, pembrolizumab, cemiplimab), antiPD-L1 (atezolizumab, durvalumab, avelumab), and anti-cytotoxic T lymphocyte-associated antigen (CTLA-4) agents (ipilimumab, tremelimumab). The PD-1/PD-L1 and CTLA-4 pathways provide negative feedback to naturally downregulate appropriate immune responses and also guard against inappropriate or autoimmune responses. In a tumor, these pathways can be hijacked by the cancer cells to promote continued proliferation and survival of the neoplastic cells. T cells found in the tumor microenvironment are frequently exhausted and ineffective against the tumor. ${ }^{1}$ These exhausted $\mathrm{T}$ cells exhibit increased expression of inhibitory receptors, such as PD-1 and CTLA-4, and also have decreased secretion of stimulatory cytokines, such as interleukin-2.,3 Tumor cell expression of PD-L1 engages the PD-1 receptor on $\mathrm{T}$ cells, promoting a shift toward inhibition and exhaustion. Interference with these inhibitory pathways using ICIs can result in restoration of tumor-directed immune responses, and ICIs have shown clinical benefit in several cancer types. ICI agents are now FDAapproved for the treatment of multiple metastatic solid tumors, including melanoma, non-small cell lung cancer (NSCLC), urothelial cancer, and many other malignancies, and are increasingly being investigated as perioperative or consolidation regimens for many of these cancers.

ICIs have an adverse effect profile that often mimics the phenotype of autoimmune disorders. Mechanistically, it has been presumed that ICI adverse events are a direct result of removing the physiologic inhibition that is preventing subclinical autoreactivity and excessive immune responses. Patients with autoimmune disease have historically been excluded from most ICI clinical trials because of the potential for increased toxicity. There are also concerns that ICIs will have decreased efficacy in these patients because of baseline immunosuppression. It is unknown, however, whether these concerns are justified based on data; the actual mechanism of ICI-induced toxicities remains poorly defined, as does 
the exact mechanism of several autoimmune disorders. Perhaps patients with underlying autoimmune disorders would have a greater benefit from ICIs because of a propensity for immune stimulation. In patients with allogeneic stem cell transplants, a small amount of graft-versus-host disease (GVHD) is desirable to ensure graft-versus-leukemia effect; there have been early encouraging results with ipilimumab in the setting of posttransplant extramedullary relapse. ${ }^{4}$ In melanoma, patients treated with PD-1 inhibitors (nivolumab or pembrolizumab) who had cutaneous immunotherapyrelated adverse events (irAEs), particularly vitiligo, did have a significantly longer overall survival, ${ }^{5,6}$ and in patients with NSCLC who initially had no response to ICI but developed ICI toxicity, resuming ICIs after resolution of irAEs could convert a number of patients to objective responders. ${ }^{7}$ It is not clear, however, that this translates to all tumor types treated with ICIs. This article reviews the application of ICI therapy to patients with solid tumors and preexisting autoimmune disorders. We summarize the current knowledge around the pathophysiology of irAEs, provide an overview of case series to date on patients with preexisting autoimmune disorders receiving ICIs in the context of solid tumors, and discuss expert advice on considering ICI therapy in the context of preexisting autoimmune disorders.

\section{Pathophysiology of irAEs and Autoimmune Disorders}

\section{Common Manifestations of irAEs}

irAEs related to ICIs are thought to be the result of an autoreactive immune response against nonmalignant tissues. Although this can result in clinical manifestations similar to those of cytotoxic chemotherapy, such as elevated transaminases, irAEs are mechanistically distinct. irAEs may not resolve from holding the ICI alone and require prompt recognition and potential intervention with steroids and/or additional immunosuppression to prevent morbidity and mortality. Common sites of irAEs include the skin, liver, thyroid, and gastrointestinal tract, but any organ system can be affected, and patients may experience multiple irAEs. Discontinuation of ICI therapy and prompt institution of immunosuppression with systemic steroids usually addresses most moderate to severe irAEs, but occasionally the addition of other immunosuppressive agents, such as infliximab or mycophenolate mofetil, is needed. ${ }^{8,9}$ Rarely, irAEs can target the muscle, heart, or central nervous system, and these systems are generally associated with a higher rate of steroid-refractoriness and mortality. ${ }^{10,11}$ A more comprehensive consideration of irAE prevalence, diagnosis, and treatment is provided in the recent update to the NCCN Clinical Practice Guidelines in Oncology (NCCN
Guidelines) for Management of Immunotherapy-Related Toxicities ${ }^{12}$ and the review by Michot et al. ${ }^{13}$

\section{Common Themes in PD-1, PD-L1, and CTLA-4}

Between Autoimmunity and irAEs

There are currently no clinically useful biomarkers that preemptively identify patients who will experience severe irAEs. This lack of predictability adds to the uncertainty of considering ICI therapy for patients with known or presumed autoimmune disease. Current understanding of the pathophysiology of autoimmunity suggests that both PD-1/PD-L1 and CTLA-4 pathways can be important in these disorders. Preclinical mouse models have shown a role for the PD-1 pathway in the pathogenesis of inflammatory bowel disease, rheumatoid arthritis (RA), autoimmune myocarditis, and rheumatological disorders, such as systemic lupus erythematosus (SLE) ${ }^{14}$ Polymorphisms in CTLA-4/CD86 have been associated with autoimmune hepatitis, Graves' disease, increased risk for acute GVHD after allogeneic stem cell transplant, and increased incidence of a variety of different cancers including leukemia. ${ }^{15-19}$ Similarly, certain polymorphisms in PD- 1 have been associated with increased risk of acute GVHD and RA. ${ }^{20,21}$ DNA methylation of genes involved in PD-1/ PD-L1 expression and the interferon-gamma pathway have also been implicated in both ICI response and susceptibility to SLE. ${ }^{22-24}$ It has also been noted that CTLA-4 is expressed on some normal tissues, such as the pituitary gland, and that direct binding of the ICI to this receptor followed by a complement-mediated immune response may also result in organ-specific toxicity. ${ }^{25,26}$

\section{Additional Shared Features Between \\ Autoimmunity and irAEs}

Common shared features between autoimmunity and irAEs are not restricted to direct links to PD-1/PD-L1 and CTLA-4 receptors. Autoreactive antibodies are also commonly associated with autoimmune disorders, such as Graves' disease, myasthenia gravis, and SLE, and have also been associated with ICI toxicity, particularly with endocrinopathies, such as hypothyroidism and hypophysitis. ${ }^{26,27}$ There also seems to be a role for autoreactive $\mathrm{T}$ cells in both autoimmunity and irAEs. $T$ cells that target tumor antigens may have homology for other off-target antigens that are present in normal tissues. This has been shown in the pmel-1 CTLA- $4^{-1-}$ mouse model, which has T cells engineered to target an antigen in melanoma and a deficiency in the CTLA-4 receptor. These mice develop vitiligo in a manner similar to that seen in some patients with melanoma treated with ipilimumab (CTLA-4 inhibitor), which suggests potential antigen cross-reactivity. ${ }^{28}$ 
It has also been hypothesized that in ICI-induced myocarditis, patients have $\mathrm{T}$ cells with specificity to a tumor antigen that has homology to muscle antigens, such as desmin or troponin. ${ }^{29}$ Given these similarities, it is reasonable to be concerned that an ICI could exacerbate an underlying autoimmune disorder.

\section{Timing of Severe irAEs}

In patients without autoimmunity, anti-PD-1/PD-L1 agents and CTLA-4 inhibitors are associated with some degree of toxicity in most patients, with up to one-third experiencing serious (grade 3/4) toxicity. Some patients who experience serious irAEs are able to resume ICI therapy after a temporary hold, but many require permanent discontinuation. Ipilimumab monotherapy (at $3 \mathrm{mg} / \mathrm{kg}$ ) is associated with a higher rate of permanent discontinuation (16\%) compared with monotherapy with anti-PD-1/PD-L1 agents $(\leq 12 \%)$ (Table 1). ${ }^{30-38}$ The combination of PD- 1 and CTLA-4 blockade results in much higher rates of grade $3 / 4$ toxicity $(46 \%-59 \%)$, but also results in higher rates of response. Fatal events related to ICI toxicity are rare, but tend to occur relatively early in the disease course; a meta-analysis of fatal events noted that the median time to fatal irAE onset was 15 days after ICI initiation. ${ }^{39}$ Based on this, it seems reasonable to hypothesize that patients who develop early and severe toxicity had an underlying predisposition, which again raises concerns about giving these agents to patients with a known predisposition to autoimmunity. However, as discussed herein, emerging evidence shows that ICIs may be reasonably safe in some patients with autoimmune disease.

\section{Literature Experience With ICls in Patients With Autoimmune Disorders}

Because of concerns about potential toxicity and compromised efficacy, patients with preexisting autoimmunity have been largely excluded from prospective randomized trials, such as those summarized in Table 1. Available information comes mostly from retrospective case series, which reflect selection and confounding biases toward relatively lower risk autoimmune disorders. Table 2 summarizes the 8 largest retrospective case series that specifically evaluated patients with preexisting autoimmunity who underwent treatment with ICIs. ${ }^{39-47}$

Most of the case series evaluated PD-1/PD-L1 blockade, but 4 included patients treated with ipilimumab. ${ }^{40,44,46,47}$ No patients in these series received dual blockade with ipilimumab/nivolumab. These case series have attempted to differentiate between an exacerbation or flare of the patient's known underlying autoimmune disorder versus a de novo irAE. The patients in these case series had a wide variety of autoimmune disorders, making it difficult to draw conclusions about safety in regard to a specific disorder. This variety does allow a few attempted generalizations about the use of ICIs in the setting of autoimmunity, however. The patients in these studies had well-controlled autoimmune disorders, and the rate of baseline systemic immunosuppression ranged from $16 \%$ to $44 \%$ in all but 1 study. ${ }^{40-46}$ irAEs and autoimmune exacerbations occurred in a minority of patients. Up to $42 \%$ of patients in the largest of the case series developed a flare of their known underlying autoimmune disorder, ${ }^{40}$ but unrelated irAEs were also seen. A number of patients developed neither a flare of

Table 1. irAEs in Phase III Trials Using ICls in Locally Advanced or Metastatic Solid Tumors

\begin{tabular}{|lllllcr|}
\hline Study & Na & Tumor & Agent & ORR & $\begin{array}{c}\text { All Grades } \\
\text { irAE Rate }\end{array}$ & $\begin{array}{c}\text { Rate of Permanent } \\
\text { Discontinuation } \\
\text { irAE Rate }\end{array}$ \\
Due to Toxicity
\end{tabular}

Abbreviations: $\mathrm{ICl}$, immune checkpoint inhibitor; irAE, immunotherapy-related adverse event; NSCLC, non-small cell lung cancer; ORR, objective response rate; $\mathrm{RCC}$, renal cell carcinoma.

aNumber of patients in the trial, none of which had autoimmune disease.

bercentage of the intention-to-treat population. 


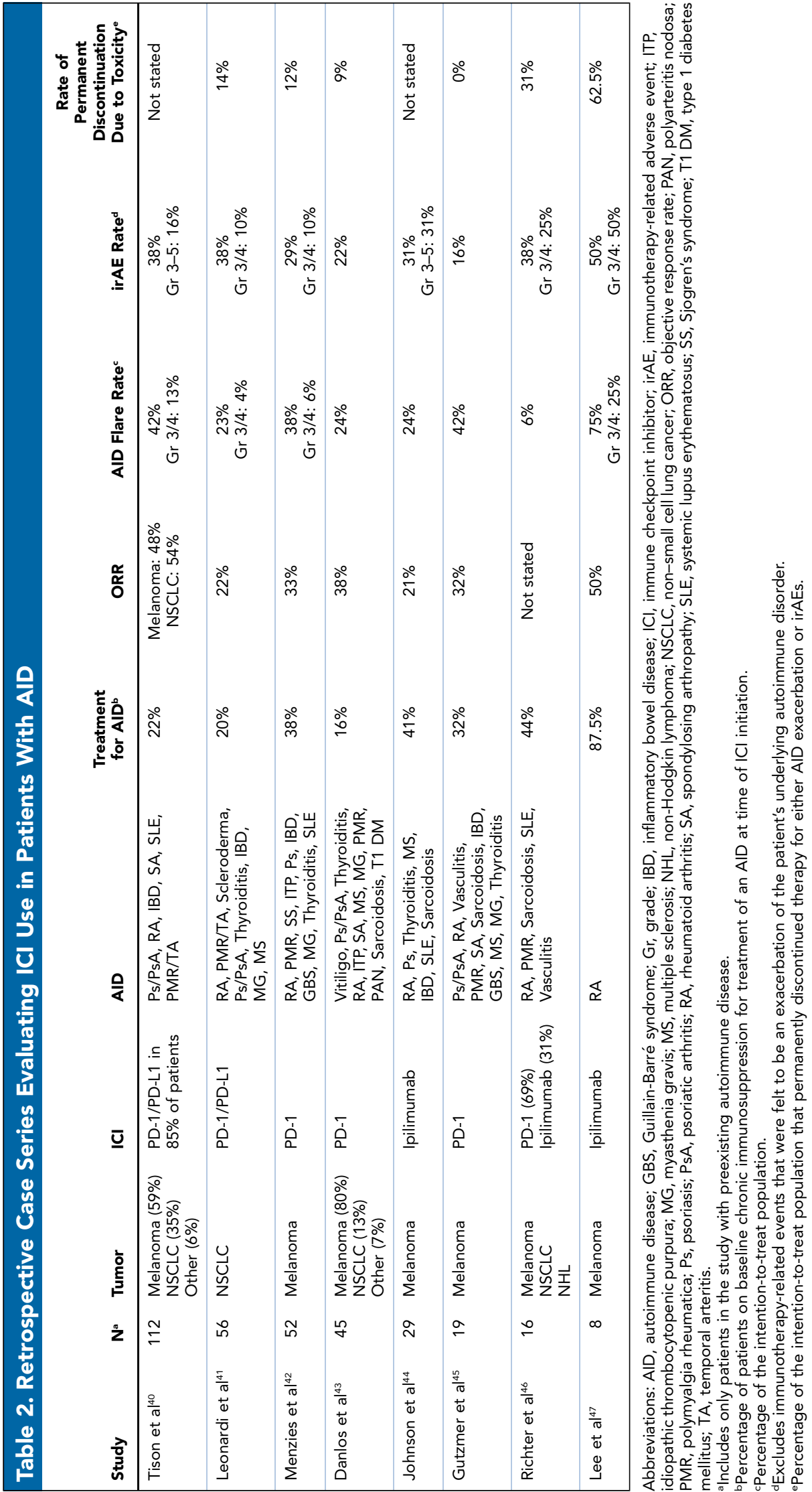


the underlying autoimmune disorder nor a new unrelated irAE. This suggests that although PD-1/PD-L1 and CTLA-4 interactions play a role in the pathogenesis of autoimmunity, there are likely other factors that contribute to both autoimmune conditions and the development of irAEs.

\section{CTLA-4 Inhibitor Experience in Patients}

\section{With Autoimmunity}

Most case series evaluated PD-1/PD-L1 inhibitors rather than CTLA- 4 inhibitors. The 2 case series that exclusively evaluated patients with autoimmunity receiving ipilimumab (anti-CTLA-4) were notable for relatively higher rates of irAEs. All patients in the ipilimumab case series who developed irAEs had grade 3-5 (severe) irAEs, ${ }^{44,47}$ and the Mayo Clinic case series in which a significant proportion of patients were treated with ipilimumab also showed a greater frequency of severe toxicity. ${ }^{46}$ Permanent discontinuation of ICIs due to toxicity was also higher in the ipilimumab case series. The largest ipilimumab case series by Johnson et $\mathrm{al}^{44}$ did not note a rate of permanent discontinuation due to toxicity, but the smaller case series had high rates of discontinuation. ${ }^{46,47}$ The ipilimumab case series were notable for a significant proportion of patients being on baseline systemic immunosuppressive therapies at the time of ipilimumab initiation, suggesting more severe autoimmune disorders, and this arguably could have adversely influenced the rate of ipilimumab discontinuation.

\section{PD-1/PD-L1 Inhibitors in Patients}

\section{With Autoimmunity}

Compared with the ipilimumab series, the PD-1/PD-L1 studies $^{40-43,45}$ reported rates of toxicity and permanent discontinuation that are comparable to those associated with use of these agents in patients without autoimmune disease. However, most patients in the case series with PD-1 inhibitors had autoimmune disorders such as RA, psoriasis, and thyroiditis; these series included significantly smaller numbers (generally 1-2) of patients with other autoimmune disorders, such as SLE, myasthenia gravis, and vasculitis. Although RA and psoriasis can be associated with significant morbidity, exacerbations of these disorders are generally not associated with a high rate of mortality. By contrast, disorders such as myasthenia gravis may be life-threatening. Myasthenia gravis that develops as an irAE in patients without preexisting autoimmunity usually requires prolonged hospitalization for management and has a relatively high rate of mortality. ${ }^{10}$ For patients with preexisting myasthenia gravis, multiple case reports describe ICIs exacerbating the condition and resulting in prolonged hospitalization for management of respiratory failure and the sequelae of neuromuscular weakness. ${ }^{48-50}$ In patients with potentially life-threatening autoimmunity, ICI therapy may be hazardous. By comparison, patients with disorders such as RA and thyroiditis may be considered for ICI therapy; only a minority develop toxicity or autoimmune exacerbation requiring immunosuppressive treatment.

\section{Patients With Autoimmunity on Baseline Immunosuppression and ICls}

There have been concerns that baseline immunosuppression at the time of ICI initiation or the need for immunosuppression to treat an irAE may decrease the efficacy of ICIs. A retrospective analysis of ICI therapy in patients with NSCLC with a baseline dose of $\geq 10 \mathrm{mg} / \mathrm{d}$ of prednisone revealed lower response rates, progressionfree survival, and overall survival compared with those treated with $<10 \mathrm{mg} / \mathrm{d}$ of prednisone..$^{51}$ Indications for prednisone in this study predominantly included fatigue, anorexia, dyspnea, and brain metastases, and the autoimmune population was not specifically considered. Patients with autoimmunity are frequently undergoing baseline treatment with steroids or other immunomodulatory agents. In the case series by Menzies et $\mathrm{al},{ }^{42} \mathrm{a}$ trend toward more frequent autoimmune exacerbations was noted in patients receiving immunosuppression for an autoimmune condition at the time of ICI initiation. It was also noted that these patients were less likely to experience response to ICI. A similar trend was noted by Tison et al, ${ }^{40}$ who reported lower progression-free and overall survival in patients receiving baseline immunosuppression.

Regarding the use of steroids for treatment of irAEs, a retrospective analysis of patients with melanoma without autoimmune disease who developed irAEs with ipilimumab and received steroids did not show any deleterious impact on ICI efficacy. ${ }^{52}$ In Tison et al, ${ }^{40}$ patients with an irAE or autoimmune exacerbation had longer progression-free and overall survival, but the initiation of steroids for treatment reversed this benefit. However, Leonardi et al ${ }^{41}$ reported no correlation between steroid initiation for toxicity and progression-free or overall survival, and 3 of the other case series noted no correlation between development of irAE or autoimmune exacerbation and disease response. ${ }^{42,45,53}$

\section{Initiation of ICI Therapy in the Autoimmune Population: Recommendations}

Based on the limited safety data from the case series, NCCN has made recommendations regarding which patients may be appropriate for considering ICI therapy in the setting of preexisting autoimmune disease. ${ }^{12}$ Patients with neurologic autoimmune disease, such as myasthenia gravis, or life-threatening autoimmune disease may not be considered good candidates for ICI therapy. Patients receiving high levels of immunosuppression for autoimmune disease control should also be approached with caution; these patients may be very difficult to manage if and when they develop toxicity, and, as discussed earlier, high doses of 


\section{Table 3. Patients With Preexisting Autoimmune Disease and Cancer}

\begin{tabular}{|ll|}
\hline ICls May Be Considered & Avoid ICls \\
\hline 1. Consult with appropriate autoimmune subspecialist & 1. Autoimmune neurologic or neuromuscular disease \\
\hline $\begin{array}{l}\text { 2. Low level of or no immunosuppression with good control of underlying } \\
\text { autoimmune disorder }\end{array}$ & 2. Life-threatening autoimmune disease \\
$\begin{array}{l}\text { 3. Patient informed consent } \\
\text { 3. Patients with poor control of autoimmune disease OR requiring high doses } \\
\text { of immunosuppressants for control }\end{array}$ \\
\hline
\end{tabular}

Abbreviation: $\mathrm{ICl}$, immune checkpoint inhibitor.

immunosuppression at the time of ICI initiation may compromise ICI efficacy.

Potential patients with preexisting autoimmune disease who could be considered for ICI treatment include those with a non-life-threatening autoimmune disease who have good control on either no immunosuppression or relatively low levels of immunosuppressive therapy (Table 3). We recommend involvement of a multidisciplinary team that includes the patient's autoimmune disease specialist in the decision to initiate ICI therapy. The autoimmune disease specialist can provide an expert opinion on the mechanisms of the underlying autoimmune toxicity, relative control and severity of the patient's underlying autoimmune disorder, assistance in tapering immunosuppression, and recommendations on the management of an autoimmune exacerbation. Avoiding systemic immunosuppression by choosing more targeted treatment of the autoimmune disorder, such as vedolizumab for inflammatory bowel disease, may allow patients to receive ICI therapy while still maintaining good control of the underlying autoimmune condition. ${ }^{54}$

The case series data favor use of PD-1/PD-L1 targeted therapy over CTLA-4-targeted therapy in patients with underlying autoimmunity; patients with autoimmune disease who received ipilimumab had a higher frequency of moderate to severe irAEs. ${ }^{44,47}$ We also recommend avoiding dual blockade with a combination of PD-(L)1/CTLA-4 agents or high-dose (10 mg/kg) ipilimumab because of much higher rates of toxicity in the general patient population (Table 1). The patient's underlying autoimmune predisposition and the irAE profile of a given ICI could also be considered; however, more data are needed for this assessment. ${ }^{39}$ Evaluation of the broader family history may unveil potential genetic predisposition to autoimmune conditions and irAEs. Finally, we recommend careful assessment of the potential risks of irAEs versus the potential benefits of treating malignancy for each individual patient. The risk/ benefit ratio of ICI therapy in patients with autoimmunity may be less favorable in the adjuvant setting..$^{53,55,56}$

Future research into the mechanisms and etiology of autoimmunity and tumor-directed immune response will provide guidance for minimizing ICI toxicity while maximizing therapeutic benefit. Allogeneic stem cell transplant for hematologic malignancies illustrates the importance of immune cell recognition of self-antigens for effective cancer therapy. Strong immune response to self-antigens may lead to GVHD, but no response to selfantigens results in loss of graft-versus-leukemia effect. ${ }^{57}$ In the same way, we must develop a balance of ICI toxicity with efficacy, because some degree of self-antigen recognition is likely needed for tumor response. Given the increasing use of ICI for a variety of tumor types, future prospective trials specifically evaluating patients with known or presumed autoimmunity may provide a more comprehensive perspective on the safety of ICIs in this population.

\section{Conclusions}

Preexisting autoimmune disorders are not an absolute contraindication to ICI therapy, but use of ICIs in these patients merits a thoughtful approach. A multidisciplinary discussion should occur between the oncologist, autoimmune specialists, and other team providers. A balance of the risks and benefits to the patient, the availability of alternative therapies, and patient preferences should be considered. Finally, it is important to continue to expand understanding of the pathogenesis of irAEs and improve the ability to predict and manage irAEs. Enrollment in prospective registries, databases, and biorepositories and use of interdisciplinary irAE tumor boards will allow clinicians to better capture, analyze, and disseminate comprehensive data that will inform clinical decisions regarding the safety and efficacy of ICI therapy for a given patient.

\footnotetext{
Submitted December 21, 2018; accepted for publication April 12, 2019.
}

Disclosures: Dr. Bhatia has disclosed that he serves on the scientific advisory board for EMD-Serono, Sanofi-Genzyme, and Bristol-Myers Squibb, and that he receives grant/research support from EMD-Serono, Merck \& Co., Inc., BristolMyers Squibb, NantKwest, Novartis, and Immune Design. Dr. Grivas has disclosed that he has received consulting fees/honoraria from Bayer, Merck \& Co., Pfizer, Bristol-Myers Squibb, AstraZeneca, Clovis Oncology, EMD Serono, Driver Inc., QED Therapeutics, Heron Therapeutics, and Janssen, and he has received grant/research support from AstraZeneca, Pfizer, Clovis Oncology, Bavarian Nordic, and Immunomedics. The remaining authors have disclosed that they have no financial interests, arrangements, affiliations, or commercial interests with the manufacturers of any products discussed in this article or their competitors.

Correspondence: Petros Grivas, MD, PhD, Department of Medicine, Division of Oncology, University of Washington, 825 Eastlake Avenue East, G-4830, Seattle, WA 98109. Email: pgrivas@uw.edu 


\section{References}

1. Thommen DS, Schumacher TN. T cell dysfunction in cancer. Cancer Cell 2018;33:547-562

2. Jiang Y, Li Y, Zhu B. T-cell exhaustion in the tumor microenvironment. Cell Death Dis 2015;6:e1792.

3. Rowshanravan B, Halliday N, Sansom DM. CTLA-4: a moving target in immunotherapy. Blood 2018;131:58-67.

4. Davids MS, Kim HT, Bachireddy $P$, et al. Ipilimumab for patients with relapse after allogeneic transplantation. N Engl J Med 2016;375:143-153.

5. Lo JA, Fisher DE, Flaherty KT. Prognostic significance of cutaneous adverse events associated with pembrolizumab therapy. JAMA Oncol 2015; 1:1340-1341.

6. Freeman-Keller M, Kim Y, Cronin $\mathrm{H}$, et al. Nivolumab in resected and unresectable metastatic melanoma: characteristics of immune-related adverse events and association with outcomes. Clin Cancer Res 2016;22: 886-894.

7. Santini FC, Rizvi H, Plodkowski AJ, et al. Safety and efficacy of re-treating with immunotherapy after immune-related adverse events in patients with NSCLC. Cancer Immunol Res 2018;6:1093-1099.

8. Brahmer JR, Lacchetti C, Schneider BJ, et al. Management of immunerelated adverse events in patients treated with immune checkpoint inhibitor therapy: American Society of Clinical Oncology clinical practice guideline. J Clin Oncol 2018;36:1714-1768.

9. Puzanov I, Diab A, Abdallah K, et al. Managing toxicities associated with immune checkpoint inhibitors: consensus recommendations from the Society for Immunotherapy of Cancer (SITC) Toxicity Management Working Group. J Immunother Cancer 2017;5:95

10. Anquetil C, Salem JE, Lebrun-Vignes B, et al. Immune checkpoint inhibitor-associated myositis. Circulation 2018;138:743-745.

11. Cuzzubbo S, Javeri F, Tissier M, et al. Neurological adverse events associated with immune checkpoint inhibitors: review of the literature. Eur J Cancer 2017;73:1-8.

12. Thompson JA, Schneider BJ, Brahmer J, et al. NCCN Clinical Practice Guidelines in Oncology: Management of Immunotherapy-Related Toxicities, Version 1.2019. To view the most recent version, visit NCCN.org. J Natl Compr Canc Netw 2019;17:255-289.

13. Michot JM, Bigenwald C, Champiat S, et al. Immune-related adverse events with immune checkpoint blockade: a comprehensive review. Eur J Cancer 2016;54:139-148.

14. Zamani MR, Aslani S, Salmaninejad A, et al. PD-1/PD-L and autoimmunity: a growing relationship. Cell Immunol 2016;310:27-41.

15. Chaouali M, Carvalho A, Tezeghdenti A, et al. Cytotoxic T lymphocyte antigen-4 gene polymorphisms and susceptibility to type 1 autoimmune hepatitis in the Tunisian population. Genes Dis 2017;5:256-262.

16. Fang M, Huang W, Mo D, et al. Association of five SNPs in cytotoxic T-lymphocyte antigen 4 and cancer susceptibility: evidence from 67 studies. Cell Physiol Biochem 2018;47:414-427.

17. Ramzi M, Arandi N, Saadi MI, et al. Genetic variation of costimulatory molecules, including cytotoxic T-lymphocyte antigen 4 , inducible T-cell costimulator, cluster differentiation 28 , and programmed cell death 1 genes, in Iranian patients with leukemia [published online April 26, 2018] Exp Clin Transplant. doi: 10.6002/ect.2017.0176

18. Karabon L, Markiewicz M, Chrobot $\mathrm{K}$, et al. The influence of genetic variations in the CD86 gene on the outcome after allogeneic hematopoietic stem cell transplantation. J Immunol Res 2018;2018:3826989.

19. Fouad NA, Saeed AM, Mahedy AW. Association of CTLA-4 +49 A/G and CT60 gene polymorphism with Graves' disease. Egypt J Immunol 2017; 24:63-70.

20. Santos N, Rodríguez-Romanos R, de la Cámara R, et al. PD-1 genotype of the donor is associated with acute graft-versus-host disease after HLAidentical sibling donor stem cell transplantation. Ann Hematol 2018;97: 2217-2224

21. Zou Y, Zhang Z, Liu Y, et al. Are programmed cell death 1 gene polymorphisms correlated with susceptibility to rheumatoid arthritis?: a metaanalysis. Medicine (Baltimore) 2017;96:e7805

22. Gao J, Shi LZ, Zhao H, et al. Loss of IFN- $\gamma$ pathway genes in tumor cells as a mechanism of resistance to anti-CTLA-4 therapy. Cell 2016;167: 397-404.e9

23. Joseph S, George NI, Green-Knox B, et al. Epigenome-wide association study of peripheral blood mononuclear cells in systemic lupus erythematosus: identifying DNA methylation signatures associated with interferon-related genes based on ethnicity and SLEDAI. J Autoimmun 2019;96:147-157.
24. Kowanetz M, Zou W, Gettinger SN, et al. Differential regulation of PD-L1 expression by immune and tumor cells in NSCLC and the response to treatment with atezolizumab (anti-PD-L1). Proc Natl Acad Sci USA 2018 115:E10119-10126

25. Caturegli P, Di Dalmazi G, Lombardi M, et al. Hypophysitis secondary to cytotoxic T-lymphocyte-associated protein 4 blockade: insights into pathogenesis from an autopsy series. Am J Pathol 2016;186:3225-3235.

26. Iwama S, De Remigis A, Callahan MK, et al. Pituitary expression of CTLA-4 mediates hypophysitis secondary to administration of CTLA-4 blocking antibody. Sci Transl Med 2014;6:230ra45

27. Osorio JC, Ni A, Chaft JE, et al. Antibody-mediated thyroid dysfunction during T-cell checkpoint blockade in patients with non-small-cell lung cancer. Ann Oncol 2017;28:583-589.

28. Byrne EH, Fisher DE. Immune and molecular correlates in melanoma treated with immune checkpoint blockade. Cancer 2017;123:2143-2153.

29. Johnson DB, Balko JM, Compton ML, et al. Fulminant myocarditis with combination immune checkpoint blockade. N Engl J Med 2016;375: 1749-1755.

30. Borghaei H, Paz-Ares L, Horn L, et al. Nivolumab versus docetaxel in advanced nonsquamous non-small-cell lung cancer. N Engl J Med 2015; 373:1627-1639.

31. Wolchok JD, Chiarion-Sileni $\mathrm{V}$, Gonzalez R, et al. Overall survival with combined nivolumab and ipilimumab in advanced melanoma. N Engl J Med 2017;377:1345-1356.

32. Robert C, Schachter J, Long GV, et al. Pembrolizumab versus ipilimumab in advanced melanoma. N Engl J Med 2015;372:2521-2532.

33. Reck M, Rodríguez-Abreu D, Robinson AG, et al. Pembrolizumab versus chemotherapy for PD-L1-positive non-small-cell lung cancer. N Engl J Med 2016;375:1823-1833.

34. Rittmeyer A, Barlesi F, Waterkamp D, et al. Atezolizumab versus docetaxe in patients with previously treated non-small-cell lung cancer (OAK): a phase 3, open-label, multicentre randomised controlled trial. Lancet 2017;389:255-265.

35. Motzer RJ, Escudier B, McDermott DF, et al. Nivolumab versus everolimus in advanced renal-cell carcinoma. N Engl J Med 2015;373: 1803-1813.

36. Powles T, Durán I, van der Heijden MS, et al. Atezolizumab versus chemotherapy in patients with platinum-treated locally advanced or metastatic urothelial carcinoma (IMvigor211): a multicentre, open-label, phase 3 randomised controlled trial. Lancet 2018;391:748-757.

37. Bellmunt J, de Wit R, Vaughn DJ, et al. Pembrolizumab as second-line therapy for advanced urothelial carcinoma. N Engl J Med 2017;376: 1015-1026.

38. Motzer RJ, Tannir NM, McDermott DF, et al. Nivolumab plus ipilimumab versus sunitinib in advanced renal-cell carcinoma. N Engl J Med 2018;378: 1277-1290.

39. Wang DY, Salem JE, Cohen JV, et al. Fatal toxic effects associated with immune checkpoint inhibitors: a systematic review and meta-analysis. JAMA Oncol 2018;4:1721-1728.

40. Tison A, Quere G, Misery L, et al. OP0196 safety and efficacy of immune checkpoint inhibitors in patients with cancer and preexisting autoimmune diseases: a nationwide multicenter retrospective study [abstract]. Ann Rheum Dis 2018;77:Abstract 147.

41. Leonardi GC, Gainor JF, Altan M, et al. Safety of programmed death-1 pathway inhibitors among patients with non-small-cell lung cancer and preexisting autoimmune disorders. J Clin Oncol 2018;36:1905-1912.

42. Menzies AM, Johnson DB, Ramanujam S, et al. Anti-PD-1 therapy in patients with advanced melanoma and preexisting autoimmune disorders or major toxicity with ipilimumab. Ann Oncol 2017;28:368-376.

43. Danlos FX, Voisin AL, Dyevre $V$, et al. Safety and efficacy of antiprogrammed death 1 antibodies in patients with cancer and pre-existing autoimmune or inflammatory disease. Eur J Cancer 2018;91:21-29.

44. Johnson DB, Sullivan RJ, Ott PA, et al. Ipilimumab therapy in patients with advanced melanoma and preexisting autoimmune disorders. JAMA Oncol 2016;2:234-240.

45. Gutzmer R, Koop A, Meier F, et al. Programmed cell death protein-1 (PD-1) inhibitor therapy in patients with advanced melanoma and preexisting autoimmunity or ipilimumab-triggered autoimmunity. Eur $\mathrm{J}$ Cancer 2017;75:24-32.

46. Richter MD, Pinkston $O$, Kottschade LA, et al. Brief report: cancer immunotherapy in patients with preexisting rheumatic disease: the Mayo Clinic experience. Arthritis Rheumatol 2018;70:356-360. 
47. Lee $B$, Wong $A$, Kee $D$, et al. The use of ipilimumab in patients with rheumatoid arthritis and metastatic melanoma. Ann Oncol 2016;27: 1174-1177.

48. Mitsune A, Yanagisawa S, Fukuhara T, et al. Relapsed myasthenia gravis after nivolumab treatment. Intern Med 2018;57:1893-1897.

49. Derle E, Benli S. Ipilimumab treatment associated with myasthenic crises and unfavorable disease course. Neurol Sci 2018;39:1773-1774.

50. Cooper DS, Meriggioli MN, Bonomi PD, et al. Severe exacerbation of myasthenia gravis associated with checkpoint inhibitor immunotherapy. J Neuromuscul Dis 2017;4:169-173.

51. Arbour KC, Mezquita L, Long N, et al. Impact of baseline steroids on efficacy of programmed cell death-1 and programmed death-ligand 1 blockade in patients with non-small-cell lung cancer. J Clin Oncol 2018; 36:2872-2878.

52. Horvat TZ, Adel NG, Dang TO, et al. Immune-related adverse events, need for systemic immunosuppression, and effects on survival and time to treatment failure in patients with melanoma treated with ipilimumab at Memorial Sloan Kettering Cancer Center. J Clin Oncol 2015;33:3193-3198.

53. Eggermont AM, Blank CU, Mandala M, et al. Adjuvant pembrolizumab versus placebo in resected stage III melanoma. N Engl J Med 2018;378: 1789-1801.

54. Bergqvist $\mathrm{V}$, Hertervig $\mathrm{E}$, Gedeon $\mathrm{P}$, et al. Vedolizumab treatment for immune checkpoint inhibitor-induced enterocolitis. Cancer Immunol Immunother 2017;66:581-592.

55. Antonia SJ, Villegas A, Daniel D, et al. Durvalumab after chemoradiotherapy in stage III non-small-cell lung cancer. N Engl J Med 2017; 377:1919-1929.

56. Weber J, Mandala M, Del Vecchio M, et al. Adjuvant nivolumab versus ipilimumab in resected stage III or IV melanoma. N Engl J Med 2017;377: 1824-1835

57. Warren EH, Deeg HJ. Dissecting graft-versus-leukemia from graft-versushost-disease using novel strategies. Tissue Antigens 2013;81:183-193. 